\title{
Definition of First Order Language with Arbitrary Alphabet. Syntax of Terms, Atomic Formulas and their Subterms ${ }^{1}$
}

\author{
Marco B. Caminati ${ }^{2}$ \\ Mathematics Department "G.Castelnuovo" \\ Sapienza University of Rome \\ Piazzale Aldo Moro 5, 00185 Roma, Italy
}

\begin{abstract}
Summary. Second of a series of articles laying down the bases for classical first order model theory. A language is defined basically as a tuple made of an integer-valued function (adicity), a symbol of equality and a symbol for the NOR logical connective. The only requests for this tuple to be a language is that the value of the adicity in $=$ is -2 and that its preimage (i.e. the variables set) in 0 is infinite. Existential quantification will be rendered (see [11]) by mere prefixing a formula with a letter. Then the hierarchy among symbols according to their adicity is introduced, taking advantage of attributes and clusters.

The strings of symbols of a language are depth-recursively classified as terms using the standard approach (see for example [16], definition 1.1.2); technically, this is done here by deploying the '-multiCat' functor and the 'unambiguous' attribute previously introduced in [10], and the set of atomic formulas is introduced. The set of all terms is shown to be unambiguous with respect to concatenation; we say that it is a prefix set. This fact is exploited to uniquely define the subterms both of a term and of an atomic formula without resorting to a parse tree.
\end{abstract}

MML identifier: FOMODEL1, version: $\underline{7.11 .07 \quad 4.160 .1126}$

The papers [1], [3], [18], [5], [6], [12], [10], [7], [8], [9], [19], [14], [13], [2], [17], [4], $[21],[22],[15]$, and [20] provide the terminology and notation for this paper.

We follow the rules: $m, n$ are natural numbers, $m_{1}, n_{1}$ are elements of $\mathbb{N}$, and $X, x, z$ are sets.

Let $z$ be a zero integer number. One can check that $|z|$ is zero.

\footnotetext{
${ }^{1}$ The author wrote this paper as part of his $\mathrm{PhD}$ thesis research.

${ }^{2}$ I would like to thank Marco Pedicini for his encouragement and support.
} 
Let us observe that there exists a real number which is negative and integer and every integer number which is positive is also natural.

Let $S$ be a non degenerated zero-one structure. Observe that (the carrier of $S) \backslash\{$ the one of $S\}$ is non empty.

We introduce languages-like which are extensions of zero-one structure and are systems

$\langle$ a carrier, a zero, a one, an adicity $\rangle$,

where the carrier is a set, the zero and the one are elements of the carrier, and the adicity is a function from the carrier $\backslash\{$ the one $\}$ into $\mathbb{Z}$.

Let $S$ be a language-like. The functor AllSymbolsOf $S$ is defined by:

(Def. 1) AllSymbolsOf $S=$ the carrier of $S$.

The functor Letters Of $S$ is defined as follows:

(Def. 2) LettersOf $S=(\text { the adicity of } S)^{-1}(\{0\})$.

The functor OpSymbolsOf $S$ is defined by:

(Def. 3) OpSymbolsOf $S=(\text { the adicity of } S)^{-1}(\mathbb{N} \backslash\{0\})$.

The functor RelSymbolsOf $S$ is defined by:

(Def. 4) RelSymbolsOf $S=(\text { the adicity of } S)^{-1}(\mathbb{Z} \backslash \mathbb{N})$.

The functor TermSymbolsOf $S$ is defined as follows:

(Def. 5) TermSymbolsOf $S=(\text { the adicity of } S)^{-1}(\mathbb{N})$.

The functor LowerCompoundersOf $S$ is defined as follows:

(Def. 6) LowerCompoundersOf $S=(\text { the adicity of } S)^{-1}(\mathbb{Z} \backslash\{0\})$.

The functor TheEqSymbOf $S$ is defined as follows:

(Def. 7) TheEqSymbOf $S=$ the zero of $S$.

The functor TheNorSymbOf $S$ is defined as follows:

(Def. 8) TheNorSymbOf $S=$ the one of $S$.

The functor OwnSymbols Of $S$ is defined by:

(Def. 9) OwnSymbolsOf $S=$ (the carrier of $S) \backslash\{$ the zero of $S$, the one of $S\}$.

Let $S$ be a language-like. An element of $S$ is an element of AllSymbolsOf $S$. The functor AtomicFormulaSymbolsOf $S$ is defined by:

(Def. 10) AtomicFormulaSymbolsOf $S=$ AllSymbolsOf $S \backslash\{$ TheNorSymbOf $S\}$.

The functor AtomicTerms of $S$ is defined by:

(Def. 11) AtomicTermsOf $S=(\text { LettersOf } S)^{1}$.

We say that $S$ is operational if and only if:

(Def. 12) OpSymbolsOf $S$ is non empty.

We say that $S$ is relational if and only if:

(Def. 13) RelSymbolsOf $S \backslash\{$ TheEqSymbOf $S\}$ is non empty.

Let $S$ be a language-like and let $s$ be an element of $S$. We say that $s$ is literal if and only if: 
(Def. 14) $s \in$ LettersOf $S$.

We say that $s$ is low-compounding if and only if:

(Def. 15) $s \in$ LowerCompoundersOf $S$.

We say that $s$ is operational if and only if:

(Def. 16) $s \in$ OpSymbolsOf $S$.

We say that $s$ is relational if and only if:

(Def. 17) $s \in$ RelSymbolsOf $S$.

We say that $s$ is termal if and only if:

(Def. 18) $s \in$ TermSymbolsOf $S$.

We say that $s$ is own if and only if:

(Def. 19) $s \in$ OwnSymbolsOf $S$.

We say that $s$ is of-atomic-formula if and only if:

(Def. 20) $s \in$ AtomicFormulaSymbolsOf $S$.

Let $S$ be a zero-one structure and let $s$ be an element of (the carrier of $S) \backslash\{$ the one of $S\}$. The functor TrivialArity $s$ yields an integer number and is defined by:

(Def. 21) TrivialArity $s=\left\{\begin{array}{l}-2, \text { if } s=\text { the zero of } S, \\ 0, \text { otherwise. }\end{array}\right.$

Let $S$ be a zero-one structure and let $s$ be an element of (the carrier of $S) \backslash\{$ the one of $S\}$. Then TrivialArity $s$ is an element of $\mathbb{Z}$.

Let $S$ be a non degenerated zero-one structure. The functor $S$ TrivialArity yielding a function from (the carrier of $S$ ) $\backslash$ the one of $S\}$ into $\mathbb{Z}$ is defined by:

(Def. 22) For every element $s$ of (the carrier of $S$ ) $\backslash\{$ the one of $S\}$ holds $(S$ TrivialArity $)(s)=$ TrivialArity $s$.

Let us observe that there exists a non degenerated zero-one structure which is infinite.

Let $S$ be an infinite non degenerated zero-one structure.

Observe that $(S \text { TrivialArity })^{-1}(\{0\})$ is infinite.

Let $S$ be a language-like. We say that $S$ is eligible if and only if:

(Def. 23) LettersOf $S$ is infinite and (the adicity of $S$ )(TheEqSymbOf $S$ ) $=-2$.

One can check that there exists a language-like which is non degenerated.

One can check that there exists a non degenerated language-like which is eligible.

A language is an eligible non degenerated language-like.

We follow the rules: $S, S_{1}, S_{2}$ are languages and $s, s_{1}, s_{2}$ are elements of $S$.

Let $S$ be a non empty language-like. Then AllSymbolsOf $S$ is a non empty set.

Let $S$ be an eligible language-like. Note that LettersOf $S$ is infinite.

Let $S$ be a language. 
Then LettersOf $S$ is a non empty subset of AllSymbolsOf $S$. Note that TheEqSymbOf $S$ is relational.

Let $S$ be a non degenerated language-like. Then AtomicFormulaSymbolsOf $S$ is a non empty subset of AllSymbolsOf $S$.

Let $S$ be a non degenerated language-like. Then TheEqSymbOf $S$ is an element of AtomicFormulaSymbolsOf $S$.

We now state the proposition

(1) Let $S$ be a language. Then LettersOf $S \cap$ OpSymbolsOf $S=\emptyset$ and TermSymbolsOf $S \cap$ LowerCompoundersOf $S=$ OpSymbolsOf $S$ and RelSymbolsOf $S \backslash$ OwnSymbolsOf $S=\{$ TheEqSymbOf $S\}$ and OwnSymbolsOf $S \subseteq$ AtomicFormulaSymbolsOf $S$ and RelSymbolsOf $S \subseteq$ LowerCompoundersOf $S$ and OpSymbolsOf $S \subseteq$ TermSymbolsOf $S$ and LettersOf $S \subseteq$ TermSymbolsOf $S \subseteq$ OwnSymbolsOf $S$ and OpSymbolsOf $S \subseteq$ LowerCompounders Of $S \subseteq$ AtomicFormulaSymbolsOf $S$.

Let $S$ be a language. One can verify the following observations:

* TermSymbolsOf $S$ is non empty,

* every element of $S$ which is own is also of-atomic-formula,

* every element of $S$ which is relational is also low-compounding,

* every element of $S$ which is operational is also termal,

* every element of $S$ which is literal is also termal,

* every element of $S$ which is termal is also own,

* every element of $S$ which is operational is also low-compounding,

* every element of $S$ which is low-compounding is also of-atomic-formula,

* every element of $S$ which is termal is also non relational,

* every element of $S$ which is literal is also non relational, and

* every element of $S$ which is literal is also non operational.

Let $S$ be a language. Note that there exists an element of $S$ which is relational and there exists an element of $S$ which is literal. Observe that every low-compounding element of $S$ which is termal is also operational. One can check that there exists an element of $S$ which is of-atomic-formula.

Let $s$ be an of-atomic-formula element of $S$. The functor ar $s$ yielding an element of $\mathbb{Z}$ is defined by:

(Def. 24) ar $s=($ the adicity of $S)(s)$.

Let $S$ be a language and let $s$ be a literal element of $S$. Note that ar $s$ is zero. The functor $S$-cons yielding a binary operation on (AllSymbolsOf $S)^{*}$ is defined as follows:

(Def. 25) $S$-cons $=$ the concatenation of AllSymbolsOf $S$.

Let $S$ be a language. 
The functor $S$-multiCat yields a function from ((AllSymbolsOf $\left.S)^{*}\right)^{*}$ into $(\text { AllSymbolsOf } S)^{*}$ and is defined by:

(Def. 26) $S$-multiCat $=($ AllSymbolsOf $S)$-multiCat .

Let $S$ be a language. The functor $S$-firstChar yielding a function from (AllSymbolsOf $S)^{*} \backslash\{\emptyset\}$ into AllSymbolsOf $S$ is defined as follows:

(Def. 27) $S$-firstChar $=($ AllSymbolsOf $S)$-firstChar .

Let $S$ be a language and let $X$ be a set. We say that $X$ is $S$-prefix if and only if:

(Def. 28) $X$ is AllSymbolsOf $S$-prefix.

Let $S$ be a language. Note that every set which is $S$-prefix is also

AllSymbolsOf $S$-prefix and every set which is AllSymbolsOf $S$-prefix is also $S$-prefix. A string of $S$ is an element of (AllSymbolsOf $S)^{*} \backslash\{\emptyset\}$.

Let us consider $S$. One can check that (AllSymbolsOf $S)^{*} \backslash\{\emptyset\}$ is non empty. Note that every string of $S$ is non empty.

Let us note that every language is infinite. Observe that AllSymbolsOf $S$ is infinite.

Let $s$ be an of-atomic-formula element of $S$, and let $S_{3}$ be a set. The functor $\operatorname{Compound}\left(s, S_{3}\right)$ is defined by:

(Def. 29) Compound $\left(s, S_{3}\right)=\left\{\langle s\rangle \frown S\right.$-multiCat $\left(S_{4}\right) ; S_{4}$ ranges over elements of $\left((\text { AllSymbolsOf } S)^{*}\right)^{*}: \operatorname{rng} S_{4} \subseteq S_{3} \wedge S_{4}$ is $|\operatorname{ar} s|$-element $\}$.

Let $S$ be a language, let $s$ be an of-atomic-formula element of $S$, and let $S_{3}$ be a set. Then $\operatorname{Compound}\left(s, S_{3}\right)$ is an element of $2^{\text {(AllSymbolsOf } S)^{*} \backslash\{\emptyset\}}$. The functor $S$-termsOfMaxDepth yields a function and is defined by the conditions (Def. 30).

(Def. 30)(i) $\operatorname{dom}(S$-termsOfMaxDepth $)=\mathbb{N}$,

(ii) $S$-termsOfMaxDepth(0) $=$ AtomicTermsOf $S$, and

(iii) for every natural number $n$ holds $S$-termsOfMaxDepth $(n+$ $1)=\bigcup\{$ Compound $(s, S$-termsOfMaxDepth $(n)) ; s$ ranges over of-atomicformula elements of $S: s$ is operational $\} \cup S$-termsOfMaxDepth $(n)$.

Let us consider $S$. Then AtomicTermsOf $S$ is a subset of (AllSymbolsOf $S)^{*}$.

Let $S$ be a language. The functor AllTermsOf $S$ is defined as follows:

(Def. 31) AllTermsOf $S=\bigcup \operatorname{rng}(S$-termsOfMaxDepth).

One can prove the following proposition

(2) $S$-termsOfMaxDepth $\left(m_{1}\right) \subseteq$ AllTermsOf $S$.

Let $S$ be a language and let $w$ be a string of $S$. We say that $w$ is termal if and only if:

(Def. 32) $w \in$ AllTermsOf $S$.

Let $m$ be a natural number, let $S$ be a language, and let $w$ be a string of $S$. We say that $w$ is $m$-termal if and only if: 
(Def. 33) $w \in S$-termsOfMaxDepth $(m)$.

Let $m$ be a natural number and let $S$ be a language. Note that every string of $S$ which is $m$-termal is also termal.

Let us consider $S$. Then $S$-termsOfMaxDepth is a function from $\mathbb{N}$ into $2^{{\text {(AllSymbolsOf } S)^{*}}^{*}}$. Then AllTermsOf $S$ is a non empty subset of (AllSymbolsOf $S)^{*}$. Note that AllTermsOf $S$ is non empty.

Let us consider $m$. One can verify that $S$-termsOfMaxDepth $(m)$ is non empty. Observe that every element of $S$-termsOfMaxDepth $(m)$ is non empty. Observe that every element of AllTermsOf $S$ is non empty.

Let $m$ be a natural number and let $S$ be a language. Note that there exists a string of $S$ which is $m$-termal. Observe that every string of $S$ which is 0 -termal is also 1-element.

Let $S$ be a language and let $w$ be a 0 -termal string of $S$. Observe that $S$-firstChar $(w)$ is literal.

Let us consider $S$ and let $w$ be a termal string of $S$. Note that $S$-firstChar $(w)$ is termal.

Let us consider $S$ and let $t$ be a termal string of $S$. The functor ar $t$ yielding an element of $\mathbb{Z}$ is defined as follows:

(Def. 34) $\operatorname{ar} t=\operatorname{ar} S$-firstChar $(t)$.

Next we state the proposition

(3) For every $m_{1}+1$-termal string $w$ of $S$ there exists an element $T$ of $S$-termsOfMaxDepth $\left(m_{1}\right)^{*}$ such that $T$ is $\mid \operatorname{ar} S$-firstChar $(w) \mid$-element and $w=\langle S \text {-firstChar }(w)\rangle^{\frown} S$-multiCat $(T)$.

Let us consider $S, m$. Note that $S$-termsOfMaxDepth $(m)$ is $S$-prefix.

Let us consider $S$ and let $V$ be an element of (AllTermsOf $S)^{*}$. Observe that $S$-multiCat $(V)$ is relation-like.

Let us consider $S$ and let $V$ be an element of (AllTermsOf $S)^{*}$. One can verify that $S$-multiCat $(V)$ is function-like.

Let us consider $S$ and let $p_{1}$ be a string of $S$. We say that $p_{1}$ is 0 -w.f.f. if and only if:

(Def. 35) There exists a relational element $s$ of $S$ and there exists an $\mid$ ar $s \mid$-element element $V$ of (AllTermsOf $S)^{*}$ such that $p_{1}=\langle s\rangle{ }^{\wedge} S$-multiCat $(V)$.

Let us consider $S$. Note that there exists a string of $S$ which is 0-w.f.f..

Let $p_{1}$ be a 0 -w.f.f. string of $S$. Observe that $S$-firstChar $\left(p_{1}\right)$ is relational. The functor AtomicFormulasOf $S$ is defined as follows:

(Def. 36) AtomicFormulasOf $S=\left\{p_{1} ; p_{1}\right.$ ranges over strings of $S: p_{1}$ is 0-w.f.f. $\}$.

Let us consider $S$. Then AtomicFormulasOf $S$ is a subset of (AllSymbolsOf $S)^{*} \backslash$ $\{\emptyset\}$. Note that AtomicFormulasOf $S$ is non empty. Observe that every element of AtomicFormulasOf $S$ is 0-w.f.f.. Observe that AllTermsOf $S$ is $S$-prefix. 
Let us consider $S$ and let $t$ be a termal string of $S$. The functor SubTerms $t$ yields an element of (AllTermsOf $S)^{*}$ and is defined by:

(Def. 37) SubTerms $t$ is $\mid \operatorname{ar} S$-firstChar $(t) \mid$-element and $t=\langle S \text {-firstChar }(t)\rangle^{\frown}$ $S$-multiCat(SubTerms $t$ ).

Let us consider $S$ and let $t$ be a termal string of $S$. One can verify that SubTerms $t$ is $|\operatorname{ar} t|$-element.

Let $t_{0}$ be a 0 -termal string of $S$. Note that SubTerms $t_{0}$ is empty.

Let us consider $m_{1}, S$ and let $t$ be an $m_{1}+1$-termal string of $S$. One can verify that SubTerms $t$ is $S$-termsOfMaxDepth $\left(m_{1}\right)$-valued.

Let us consider $S$ and let $p_{1}$ be a 0 -w.f.f. string of $S$. The functor SubTerms $p_{1}$ yields an $\mid \operatorname{ar} S$-firstChar $\left(p_{1}\right) \mid$-element element of $(\text { AllTermsOf } S)^{*}$ and is defined as follows:

(Def. 38) $\quad p_{1}=\left\langle S\right.$-firstChar $\left.\left(p_{1}\right)\right\rangle \frown S$-multiCat $\left(\operatorname{SubTerms} p_{1}\right)$.

Let us consider $S$ and let $p_{1}$ be a 0 -w.f.f. string of $S$. Note that SubTerms $p_{1}$ is $\mid \operatorname{ar} S$-firstChar $\left(p_{1}\right) \mid$-element.

Then AllTermsOf $S$ is an element of $2^{(\text {AllsymbolsOf } S)^{*} \backslash\{\emptyset\}}$. Note that every element of AllTermsOf $S$ is termal. The functor $S$-subTerms yielding a function from AllTermsOf $S$ into (AllTermsOf $S$ ) ${ }^{*}$ is defined by:

(Def. 39) For every element $t$ of AllTermsOf $S$ holds $S$-subTerms $(t)=$ SubTerms $t$.

We now state several propositions:

(4) $S$-termsOfMaxDepth $(m) \subseteq S$-termsOfMaxDepth $(m+n)$.

(5) If $x \in$ AllTermsOf $S$, then there exists $n_{1}$ such that $x \in$ $S$-termsOfMaxDepth $\left(n_{1}\right)$.

(6) AllTermsOf $S \subseteq(\text { AllSymbolsOf } S)^{*} \backslash\{\emptyset\}$.

(7) AllTermsOf $S$ is $S$-prefix.

(8) If $x \in$ AllTermsOf $S$, then $x$ is a string of $S$.

(9) AtomicFormulaSymbolsOf $S \backslash$ OwnSymbolsOf $S=\{$ TheEqSymbOf $S\}$.

(10) TermSymbolsOf $S \backslash$ Letters Of $S=$ OpSymbolsOf $S$.

(11) AtomicFormulaSymbolsOf $S \backslash$ RelSymbolsOf $S=$ TermSymbolsOf $S$.

Let us consider $S$. Observe that every of-atomic-formula element of $S$ which is non relational is also termal.

Then OwnSymbolsOf $S$ is a subset of AllSymbolsOf $S$. Observe that every termal element of $S$ which is non literal is also operational.

Next we state three propositions:

(12) $x$ is a string of $S$ iff $x$ is a non empty element of (AllSymbolsOf $S$ )*.

(13) $x$ is a string of $S$ iff $x$ is a non empty finite sequence of elements of AllSymbolsOf $S$.

(14) $S$-termsOfMaxDepth is a function from $\mathbb{N}$ into $2^{(\text {AllSymbolsOf } S)^{*}}$. 
Let us consider $S$. Note that every element of LettersOf $S$ is literal. One can check that TheNorSymbOf $S$ is non low-compounding.

Observe that TheNorSymbOf $S$ is non own.

Next we state the proposition

(15) If $s \neq$ TheNorSymbOf $S$ and $s \neq$ TheEqSymbOf $S$, then $s \in$ OwnSymbolsOf $S$.

For simplicity, we use the following convention: $l, l_{1}, l_{2}$ denote literal elements of $S, a$ denotes an of-atomic-formula element of $S, r$ denotes a relational element of $S, w, w_{1}$ denote strings of $S$, and $t_{2}$ denotes an element of AllTermsOf $S$.

Let us consider $S, t$. The functor Depth $t$ yielding a natural number is defined by:

(Def. 40) $t$ is Depth $t$-termal and for every $n$ such that $t$ is $n$-termal holds Depth $t \leq$ $n$.

Let us consider $S$, let $m_{0}$ be a zero number, and let $t$ be an $m_{0}$-termal string of $S$. Note that Depth $t$ is zero.

Let us consider $S$ and let $s$ be a low-compounding element of $S$. Note that ar $s$ is non zero.

Let us consider $S$ and let $s$ be a termal element of $S$. Observe that ar $s$ is non negative and extended real.

Let us consider $S$ and let $s$ be a relational element of $S$. Note that ar $s$ is negative and extended real.

Next we state the proposition

(16) If $t$ is non 0-termal, then $S$-firstChar $(t)$ is operational and SubTerms $t \neq$ $\emptyset$.

Let us consider $S$. Observe that $S$-multiCat is finite sequence-yielding.

Let us consider $S$ and let $W$ be a non empty AllSymbolsOf $S^{*} \backslash\{\emptyset\}$-valued finite sequence. One can verify that $S$-multiCat $(W)$ is non empty.

Let us consider $S, l$. Note that $\langle l\rangle$ is 0-termal.

Let us consider $S, m, n$. One can check that every string of $S$ which is $m+0 \cdot n$-termal is also $m+n$-termal.

Let us consider $S$. One can check that every own element of $S$ which is non low-compounding is also literal.

Let us consider $S, t$. One can check that SubTerms $t$ is $\operatorname{rng} t^{*}$-valued.

Let $p_{0}$ be a 0 -w.f.f. string of $S$. Observe that SubTerms $p_{0}$ is $\operatorname{rng} p_{0}{ }^{*}$-valued. Then $S$-termsOfMaxDepth is a function from $\mathbb{N}$ into $2^{\text {(AllsymbolsOf } S)^{*} \backslash\{\emptyset}$.

Let us consider $S, m_{1}$. Observe that $S$-termsOfMaxDepth $\left(m_{1}\right)$ has non empty elements.

Let us consider $S, m$ and let $t$ be a termal string of $S$. One can verify that $t$ null $m$ is Depth $t+m$-termal. One can check that every string of $S$ which is termal is also TermSymbolsOf $S$-valued. Observe that AllTermsOf $S \backslash$ (TermSymbolsOf $S)^{*}$ is empty. 
Let $p_{0}$ be a 0 -w.f.f. string of $S$. Observe that SubTerms $p_{0}$ is TermSymbolsOf $S^{*}$-valued. One can verify that every string of $S$ which is 0 w.f.f. is also

AtomicFormulaSymbolsOf $S$-valued. One can check that OwnSymbolsOf $S$ is non empty.

In the sequel $p_{0}$ is a 0 -w.f.f. string of $S$.

The following proposition is true

(17) If $S$-firstChar $\left(p_{0}\right) \neq$ TheEqSymbOf $S$, then $p_{0}$ is OwnSymbolsOf $S$ valued.

Let us observe that there exists a language-like which is strict and non empty.

Let $S_{1}, S_{2}$ be languages-like. We say that $S_{2}$ is $S_{1}$-extending if and only if:

(Def. 41) The adicity of $S_{1} \subseteq$ the adicity of $S_{2}$ and TheEqSymbOf $S_{1}=$ TheEqSymbOf $S_{2}$ and TheNorSymbOf $S_{1}=$ TheNorSymbOf $S_{2}$.

Let us consider $S$. One can verify that $S$ null is $S$-extending. Observe that there exists a language which is $S$-extending.

Let us consider $S_{1}$ and let $S_{2}$ be an $S_{1}$-extending language. Observe that OwnSymbolsOf $S_{1} \backslash$ OwnSymbolsOf $S_{2}$ is empty.

Let $f$ be a $\mathbb{Z}$-valued function and let $L$ be a non empty language-like. The functor $L$ extendWith $f$ yields a strict non empty language-like and is defined by the conditions (Def. 42).

(Def. 42)(i) The adicity of $L$ extendWith $f=f\lceil(\operatorname{dom} f \backslash\{$ the one of $L\})+$-the adicity of $L$,

(ii) the zero of $L$ extendWith $f=$ the zero of $L$, and

(iii) the one of $L$ extendWith $f=$ the one of $L$.

Let $S$ be a non empty language-like and let $f$ be a $\mathbb{Z}$-valued function. Note that $S$ extendWith $f$ is $S$-extending.

Let $S$ be a non degenerated language-like. Observe that every language-like which is $S$-extending is also non degenerated.

Let $S$ be an eligible language-like. One can check that every language-like which is $S$-extending is also eligible.

Let $E$ be an empty binary relation and let us consider $X$. Note that $X\lceil E$ is empty.

Let us consider $X$ and let $m$ be an integer number. Note that $X \longmapsto m$ is $\mathbb{Z}$-valued.

Let us consider $S$ and let $X$ be a functional set.

The functor $S$ addLettersNotIn $X$ yields an $S$-extending language and is defined as follows:

(Def. 43) $S$ addLettersNotIn $X=$

$S$ extendWith((AllSymbolsOf $S \cup$ SymbolsOf $X)$-freeCountableSet $\longmapsto$

0 qua $\mathbb{Z}$-valued function). 
Let us consider $S_{1}$ and let $X$ be a functional set.

Note that LettersOf $\left(S_{1}\right.$ addLettersNotIn $\left.X\right) \backslash$ SymbolsOf $X$ is infinite.

Let us note that there exists a language which is countable.

Let $S$ be a countable language. Observe that AllSymbolsOf $S$ is countable. One can verify that (AllSymbolsOf $S)^{*} \backslash\{\emptyset\}$ is countable.

Let $L$ be a non empty language-like and let $f$ be a $\mathbb{Z}$-valued function. Note that AllSymbolsOf $(L$ extendWith $f)-(\operatorname{dom} f \cup$ AllSymbolsOf $L)$ is empty.

Let $S$ be a countable language and let $X$ be a functional set. One can check that $S$ addLettersNotIn $X$ is countable.

\section{REFERENCES}

[1] Grzegorz Bancerek. Cardinal numbers. Formalized Mathematics, 1(2):377-382, 1990.

[2] Grzegorz Bancerek. The fundamental properties of natural numbers. Formalized Mathematics, 1(1):41-46, 1990.

[3] Grzegorz Bancerek. König's theorem. Formalized Mathematics, 1(3):589-593, 1990.

[4] Grzegorz Bancerek. The ordinal numbers. Formalized Mathematics, 1(1):91-96, 1990.

[5] Grzegorz Bancerek and Krzysztof Hryniewiecki. Segments of natural numbers and finite sequences. Formalized Mathematics, 1(1):107-114, 1990.

[6] Czesław Byliński. Finite sequences and tuples of elements of a non-empty sets. Formalized Mathematics, 1(3):529-536, 1990.

[7] Czesław Byliński. Functions and their basic properties. Formalized Mathematics, 1(1):55$65,1990$.

[8] Czesław Byliński. Functions from a set to a set. Formalized Mathematics, 1(1):153-164, 1990.

[9] Czesław Byliński. The modification of a function by a function and the iteration of the composition of a function. Formalized Mathematics, 1(3):521-527, 1990.

[10] Marco B. Caminati. Preliminaries to classical first order model theory. Formalized Mathematics, 19(3):155-167, 2011, doi: 10.2478/v10037-011-0025-2.

[11] Marco B. Caminati. First order languages: Further syntax and semantics. Formalized Mathematics, 19(3):179-192, 2011, doi: 10.2478/v10037-011-0027-0.

[12] Agata Darmochwał. Finite sets. Formalized Mathematics, 1(1):165-167, 1990.

[13] Katarzyna Jankowska. Transpose matrices and groups of permutations. Formalized Mathematics, 2(5):711-717, 1991.

[14] Rafał Kwiatek and Grzegorz Zwara. The divisibility of integers and integer relative primes. Formalized Mathematics, 1(5):829-832, 1990.

[15] Beata Padlewska. Families of sets. Formalized Mathematics, 1(1):147-152, 1990.

[16] W. Pohlers and T. Glaß. An introduction to mathematical logic. Vorlesungsskriptum, WS, 93, 1992

[17] Marta Pruszyńska and Marek Dudzicz. On the isomorphism between finite chains. Formalized Mathematics, 9(2):429-430, 2001.

[18] Andrzej Trybulec. Domains and their Cartesian products. Formalized Mathematics, $1(\mathbf{1}): 115-122,1990$

[19] Michał J. Trybulec. Integers. Formalized Mathematics, 1(3):501-505, 1990.

[20] Zinaida Trybulec. Properties of subsets. Formalized Mathematics, 1(1):67-71, 1990.

[21] Edmund Woronowicz. Relations and their basic properties. Formalized Mathematics, 1(1):73-83, 1990.

[22] Edmund Woronowicz. Relations defined on sets. Formalized Mathematics, 1(1):181-186, 1990. 